\title{
Independent and interactive effects of HIV infection, clinical stage and other comorbidities on survival of children treated for severe malnutrition in rural South Africa: A retrospective multicohort study
}

\author{
M Muzigaba, ${ }^{1,2} \mathrm{PhD}, \mathrm{MPH}$, MPhil, BSc; T Puoane, ${ }^{2}$ Dr PH, MPH, BCur, BSocSci; \\ B Sartorius, ${ }^{3} \mathrm{PhD}$, EPIET, MSc, BSc Hons, BSc; D Sanders, ${ }^{2} \mathrm{MB}$ ChB, DCH, MRCP, DTPH, DSc \\ ${ }^{1}$ School of Clinical Medicine, College of Health Sciences, University of KwaZulu-Natal, Durban, South Africa \\ ${ }^{2}$ Faculty of Community and Health Sciences, School of Public Health, University of the Western Cape, Cape Town, South Africa \\ ${ }^{3}$ Discipline of Public Health Medicine, School of Nursing and Public Health, University of KwaZulu-Natal, Durban, South Africa
}

Corresponding author: M Muzigaba (mochemoseo@gmail.com)

\begin{abstract}
Background. There is still limited to no evidence on the independent and interactive effects of HIV infection, disease stage, baseline disease severity and other important comorbidities on mortality risk among young children treated for severe acute malnutrition (SAM) in South Africa (SA, using the World Health Organization (WHO) recommended treatment modality.

Objectives. To determine baseline clinical characteristics among children with SAM and assess whether HIV infection, disease stage, critical illness at baseline and other comorbidities independently and interactively contributed to excess mortality in this sample.

Methods. We followed up children aged 6 - 60 months, who were admitted with and treated for SAM at two rural hospitals in SA, and retrospectively reviewed their treatment records to abstract data on their baseline clinical characteristics and treatment outcomes. In total, 454 children were included in the study. Descriptive statistical tests were used to summarise patients' clinical characteristics. KaplanMeier failure curves were created for key characteristics and compared statistically using log-rank tests. Univariate and multivariate Cox regression was used to estimate independent and interactive effects.

Results. The combined case fatality rate was $24.4 \%$. HIV infection, clinical disease stage, the presence of lower respiratory tract infection, marasmus and disease severity at baseline were all independently associated with excess mortality. The critical stage for higher risk of death was when cases were admitted at WHO stage III. The interactions of two or three of these characteristics were associated with increased risk of death when compared with having none, with HIV infection and critical illness showing the greatest risk (hazard ratio 22, $p<0.001$ ). Conclusion. The high HIV prevalence rate in the study setting and the resultant treatment outcomes support the notion that the WHO treatment guidelines should be revised to ensure that mechanisms for effective treatment of HIV comorbidity in SAM are in place. However, a much more rigorous study is warranted to verify this conclusion.
\end{abstract}

S Afr J Child Health 2017;11(1):46-53. DOI:10.7196/SAJCH.2017v11i1.1202

Childhood undernutrition remains a major public health concern in developing countries, and has in the past been shown to contribute to $\sim 50 \%$ of the nearly 10 million under- 5 children years who die each year of preventable causes. ${ }^{[1]}$ Although severe acute malnutrition (SAM) is seldom recognised, this condition remains an extremely widespread disorder associated with high rates of mortality and morbidity, and requires specialised treatment and interventions. ${ }^{[1]}$

In an effort to reduce deaths from SAM and improve recovery, the World Health Organization (WHO) has developed a '10-step' guideline for managing SAM. ${ }^{[2]}$ These guidelines have since been promoted as the standard treatment modality for clinical care of severely malnourished children. ${ }^{[3]}$ There is evidence to show that if implemented correctly, the protocol can improve case fatality rates (CFRs) from $\sim 40 \%$ to $<10 \%,{ }^{[4]}$ even when applied in emergency humanitarian situations. ${ }^{[5]}$ These guidelines have gained recognition worldwide and are now being used in most healthcare units, including some hospitals in South Africa (SA). ${ }^{[6]}$

While numerous studies have established the adverse effects of HIV infection on the survival of children treated for SAM ${ }^{[7-9]}$ few have studied the independent effects of HIV clinical stage in particular, as well as disease severity at baseline and other critical comorbidities on admission. There is also limited or no evidence on the interactive effects of these clinical characteristics on increased mortality risk.
This study was, in part, prompted by the lack of such evidence and the fact that in the study setting, the high CFRs for SAM were being attributed to HIV infection rather than to mismanagement of SAM children by healthcare workers. There was also some anecdotal evidence from clinicians in the same hospitals that, depending on the clinical stage of HIV infection, the WHO 10-step protocol may show no effect. This study therefore sought to establish whether there was (i) an independent effect of HIV infection, HIV clinical stage, baseline disease severity and other clinical factors on survival prospects of children admitted and treated for SAM using the WHO 10-step guidelines; and (ii) an added risk of death depending on the interaction of two or more of these baseline clinical characteristics.

\section{Methods \\ Study setting}

This study was conducted in two rural district hospitals located in the Eastern Cape Province of SA. The two hospitals were purposefully selected on the basis of being the only hospitals in the region that had shown optimal implementation of the WHO 10-step guidelines and had the basic resources to do so at the time. ${ }^{[10]}$ Furthermore, the hospitals serve catchment areas where there is a high HIV prevalence. This increased the likelihood of enrolling enough HIVpositive children with SAM for the purposes of the study. 


\section{Study design}

This study consisted of an observational study design with a prospective and retrospective component. The prospective aspect involved: (i) identifying and classifying multiple cohorts of SAM cases admitted to two different hospitals, at different study intervals, according to their nutritional and HIV status; (ii) identifying their clinical profile on admission; and (iii) initiating them on treatment and following them up to assess specific treatment outcomes. The retrospective component involved reviewing treatment records, which were compiled prospectively to document outcomes of interest, and linking this information with baseline characteristics.

\section{Study sample}

Patient treatment records were only considered for the study if they belonged to SAM patients admitted to either of the hospitals between January 2009 and May 2013 (the period during which treatment of SAM cases was enforced and monitored) and if patients were between the ages of 6 months and 5 years. The patient treatment records considered also contained clearly defined malnutrition status as per the Wellcome classification, ${ }^{[11]}$ had records showing HIV test results and HIV clinical stage, and included a complete treatment record of the child while in the hospital. The Wellcome system was more practical than other classification methods, as there were some inconsistencies in the measurement of height/length. A comprehensive written medical examination by a doctor and the discharge criteria followed for patients who did not die while being treated were also used as eligibility criteria. In total, 466 treatment records meeting the inclusion criteria were identified from both hospitals during the study period. This was $\sim 85 \%$ of the total number of treatment records that were available during the same period. The records that were selected constituted the unit of data abstraction and analysis.

\section{Patient recruitment}

This study was approved by the University of the Western Cape Research Ethics Committee (reg. no. 12/10/37). During the study period, the parents or guardians who brought their children with SAM to the hospital for treatment were approached in the ward after admission and informed about the study. They were then asked to provide informed consent for their children to be enrolled in the study. For the children whose parents provided consent, the inpatient numbers recorded on the treatment charts were noted by a research nurse for later reference during retrospective record review.

\section{Data generation and collection}

Data were extracted from patient treatment records. A structured and validated questionnaire developed by the International Malnutrition Taskforce and Muhimbili National Hospital, Dar es Salaam, Tanzania ${ }^{[12]}$ was used for extraction and collection of all the data.

SAM classification was done on admission by the admitting doctor, based on the Wellcome criteria. ${ }^{[11]}$ Confidential and private counselling for HIV testing was conducted by professionally trained nurses for all parents and guardians of children with SAM. Parents and guardians were also requested to give permission for an HIV polymerase chain reaction test to be done for their children. Parents/ guardians provided their own consent and were tested for HIV using an enzyme-linked immunosorbent assay test. HIV clinical staging was done by the admitting doctor as per the WHO guidelines. ${ }^{[13]}$ The admitting doctor also graded oedema and dermatosis on admission if they were present, and confirmed the presence of lower respiratory tract infections (LRTIs). LRTI was an umbrella term used for cases with comorbidities such as pneumonia, bronchitis and other infections below the larynx. Tuberculosis (TB) was not considered as a comorbidity in this study as the condition was mostly underdiagnosed, which resulted in too few cases being included in the study to be studied separately.
Based on diagnostic information, SAM cases were also classified as critically ill or not. Definition of a case as critically ill was based on whether or not they were admitted with one or a combination of five clinical features, i.e.: (i) depressed conscious state (prostration or coma); (ii) bradycardia; (iii) evidence of shock with or without dehydration; (iv) hypoglycaemia; and ( $v$ ) hypothermia. These clinical manifestations have been documented as the strongest predictors of early death (within 24 hours of admission). ${ }^{[14]}$ Other comorbidities (excluding LRTIs, TB and HIV/AIDS described above) directly or indirectly related to SAM were also noted, e.g. lethargy, hyponatraemia and hypokalaemia, dehydration, deep acidotic breathing, anaemia and pyrexia, herbal intoxication, diarrhoea, burns and other hereditary dysfunctions commonly reported by the doctors in each hospital. ${ }^{[14]}$ The outcomes in this study were death while under treatment or discharge following treatment completion, i.e. survival.

\section{Data analysis}

All the data were processed and analysed using Stata/IC 13.0 (StataCorp. LP, USA). Survival analysis was used to assess time to death for SAM cases based on baseline clinical characteristics. Firstly, Kaplan-Meier failure curves for key characteristics were created and compared statistically using log-rank tests. The log-rank test was performed among groups for each predictor variable to determine whether certain predictors needed to be in the final model. A predictor was included in the model if $p<0.20$. This elimination scheme, though arbitrary, allowed for the exclusion of variables that were less likely to contribute anything to the model, which included other predictors. Therefore, the unadjusted Cox proportional hazard model consisted of predictors where $p<0.20$. The predictors that were not statistically significant at univariate level were dropped from the final multivariate (adjusted) model. The Cox formulation was also used to quantify the impact of the interaction of factors on the survival prospects of study subjects, and to identify potential effect modifiers. Both the unadjusted, adjusted and interaction models were tabulated with hazard ratios (HRs), 95\% confidence intervals (CIs) and the corresponding $p$-values. Throughout the analysis, $p<0.05$ was considered statistically significant. The final model was tested to determine whether it did not violate the proportional hazard assumption.

\section{Results \\ Descriptive results}

Over the study period 466 subjects met the study inclusion criteria and were considered for the study. However, 12 SAM cases defaulted or absconded treatment and were censored during data analysis, resulting in 454 cases being analysed. The majority of subjects (50\%, $n=225$ ) were aged between 13 and 24 months, followed by cases aged 6 - 12 months $(36 \%, n=165)$. Cases aged 3 - 5 years constituted only $14 \%$ of the study sample.

Pooled data revealed that the proportion with marasmus and kwashiorkor was similar (38\% and $40 \%$, respectively), whereas only $22 \%$ were classified as marasmus-kwashiorkor (Table 1). About a quarter (28\%) of SAM cases had LRTIs on admission, and a third had other comorbidities, the most common being gastroenteritis (11\%). In both hospitals, the most commonly diagnosed LRTI was pneumonia. Pooled data also showed that 196 (43\%) were HIV-positive. In total, the majority who tested positive for HIV were classified as disease stage III (37\%), followed by those who were at stage II (26.5\%), then $19 \%$ and $17 \%$ for stages I and IV, respectively. Approximately $26 \%$ of SAM cases were admitted in a critical condition as per study definition. The combined CFR was $24.4 \%$.

Pooled analysis revealed that more HIV-positive SAM patients were marasmic compared with their HIV-negative counterparts (56\% and $24 \%$, respectively, $p<0.001$ ) (Table 2 ). The reverse was 
Table 1. Comparison of clinical characteristics of SAM cases by hospital on admission $(N=454)$

\begin{tabular}{|c|c|c|c|c|}
\hline Variable & Hospital A & Hospital B & $\begin{array}{l}\text { Both hospitals } \\
\text { combined }\end{array}$ & $p$-value ${ }^{*}$ \\
\hline Severe malnutrition classification & & & & 0.171 \\
\hline Marasmus & $59(40.9)$ & $114(36.8)$ & $173(38.1)$ & \\
\hline Kwashiorkor & $61(42.4)$ & $120(38.7)$ & $181(39.9)$ & \\
\hline Marasmus-kwashiorkor & $24(16.7)$ & $76(24.5)$ & $100(22.0)$ & \\
\hline Oedema grade & & & & 0.851 \\
\hline None & $52(36.1)$ & $101(32.6)$ & $153(33.7)$ & \\
\hline Mild & $9(6.2)$ & $20(6.4)$ & $29(6.4)$ & \\
\hline Moderate & $39(27.0)$ & $83(26.7)$ & $122(26.9)$ & \\
\hline Severe & $44(30.6)$ & $106(34.2)$ & $150(33.1)$ & \\
\hline Dermatosis grade & & & & 0.122 \\
\hline None & $36(25.0)$ & $100(32.6)$ & $136(29.9)$ & \\
\hline Mild & $43(29.8)$ & $63(20.3)$ & $106(23.4)$ & \\
\hline Moderate & $47(32.6)$ & $110(35.5)$ & $157(34.6)$ & \\
\hline Severe & $18(12.5)$ & $37(11.9)$ & $55(12.1)$ & \\
\hline LRTIs & & & & 0.156 \\
\hline Yes & $48(33.3)$ & $83(26.7)$ & $131(28.9)$ & \\
\hline No & $96(66.7)$ & $227(73.3)$ & $323(71.2)$ & \\
\hline Other comorbidities & & & & 0.140 \\
\hline Yes & $55(38.2)$ & $97(31.3)$ & $152(33.4)$ & \\
\hline No & $89(61.8)$ & $213(68.7)$ & $302(66.5)$ & \\
\hline Critically ill on admission & & & & 0.344 \\
\hline Yes & $33(22.9)$ & $84(27.1)$ & $117(25.7)$ & \\
\hline No & $111(77.1)$ & $226(72.9)$ & $337(74.2)$ & \\
\hline HIV status & & & & 0.001 \\
\hline Positive & $78(54.1)$ & $118(38.0)$ & $196(43.2)$ & \\
\hline Negative & $66(45.8)$ & $192(61.9)$ & $258(56.8)$ & \\
\hline HIV/AIDS disease stage & & & & 0.191 \\
\hline 1 & $18(23.0)$ & $16(13.6)$ & $34(17.4)$ & \\
\hline 2 & $22(28.2)$ & $30(25.4)$ & $52(26.5)$ & \\
\hline 3 & $23(29.4)$ & $50(42.7)$ & $73(37.2)$ & \\
\hline 4 & $15(19.2)$ & $22(18.6)$ & $37(18.8)$ & \\
\hline Outcome & & & & 0.960 \\
\hline Died & $34(24.3)$ & $74(24.5)$ & $108(24.4)$ & \\
\hline Discharged & $106(75.7)$ & $228(75.7)$ & $334(75.6)$ & \\
\hline
\end{tabular}

Table 2. Cross-tabulation of cases by SAM syndromic classification and HIV status: Hospital-level and pooled analyses $(2009-2013)$

\begin{tabular}{|c|c|c|c|c|}
\hline & \multicolumn{4}{|c|}{ Clinical classification of severe malnutrition, $n(\%)$} \\
\hline & Marasmus & Kwashiorkor & Marasmus-kwashiorkor & Total, $n$ \\
\hline \multicolumn{5}{|l|}{ Hospital A } \\
\hline HIV-negative & $15(22.7)$ & $41(62.1)$ & $10(15.2)$ & 66 \\
\hline HIV-positive & $44(56.4)$ & $20(25.6)$ & $14(17.9)$ & 78 \\
\hline Total & $59(40.9)$ & $61(42.3)$ & $24(16.7)$ & 144 \\
\hline \multicolumn{5}{|l|}{ Hospital B } \\
\hline HIV-negative & $48(25.0)$ & $98(51.0)$ & $46(23.9)$ & 192 \\
\hline HIV-positive & $66(55.9)$ & $22(18.6)$ & $30(25.4)$ & 118 \\
\hline Total & $114(36.8)$ & $120(38.7)$ & $76(25.5)$ & 310 \\
\hline \multicolumn{5}{|c|}{ Both hospitals combined } \\
\hline HIV-negative & $63(24.4)$ & $139(53.8)$ & $56(21.7)$ & 258 \\
\hline HIV-positive & $110(56.1)$ & $42(21.4)$ & $44(22.5)$ & 196 \\
\hline Total & $173(38.1)$ & $181(39.9)$ & $100(22.3)$ & 454 \\
\hline
\end{tabular}


true for cases who had kwashiorkor, where $53 \%$ were HIV-negative and $21 \%$ HIVpositive $(p<0.001)$. The same direction of differences was also observed at the level of individual hospitals, with a similar statistical significance $(p<0.001)$. For cases with marasmus-kwashiorkor, there were no statistically significant differences across HIV status $(p>0.05)$.

\section{Survival analysis}

\section{Univariate survival analysis}

Based on the log-rank test for equality of failure functions, mortality hazard did not differ significantly by hospital $(p=0.341)$. Hence, for subsequent analysis the hospitals were combined.

In this study, $41.15 \%$ of HIV-positive and $11.6 \%$ of HIV-negative SAM cases died while under treatment $(p<0.001)$ (Fig. 1). HIVpositive cases had worse survival prospects than their HIV-negative counterparts in both hospitals $(p<0.001)$. As shown in the graph, the cumulative fraction of HIVpositive SAM cases who had died by day 6 post admission was $25 \%$ compared with HIV-negative cases, which was about $5 \%$ at the same time point. These results imply that HIV-positive cases generally died sooner and in greater numbers following admission, than their HIV-negative counterparts. Stage IV cases had the worst survival prospects followed by their stage III counterparts. The difference between these two groups was statistically significant $(p<0.01)$ as was the difference between stage I and stage II SAM cases $(p=0.043)$ and stage II and stage III cases $(p<0.001)$. These results may imply that the critical stage for higher risk of death was when cases were admitted at stage III.

Approximately $25 \%$ of children admitted in a critical condition had died by the second day, compared with only $1 \%$ of the non-critically ill group $(p<0.001)$ (Fig. 2). Mortality patterns beyond the second day showed that $\sim 75 \%$ of critically ill children with SAM had died by the 28th day after hospitalisation, compared with about $17 \%$ who were not critically ill. Survival prospects for SAM cases who were admitted with marasmus were significantly poorer compared with cases who had either kwashiorkor or marasmic-kwashiorkor ( $p=0.001)$. No differences were found between cases that had kwashiorkor and marasmuskwashiorkor $(p=0.052)$. Furthermore, the presence of other comorbidities and coinfection with LTRIs/TB was associated with excess mortality.

\section{Cox proportional hazard model} Univariate results

The univariate results shown in Table 3 indicate that only HIV status and stage,

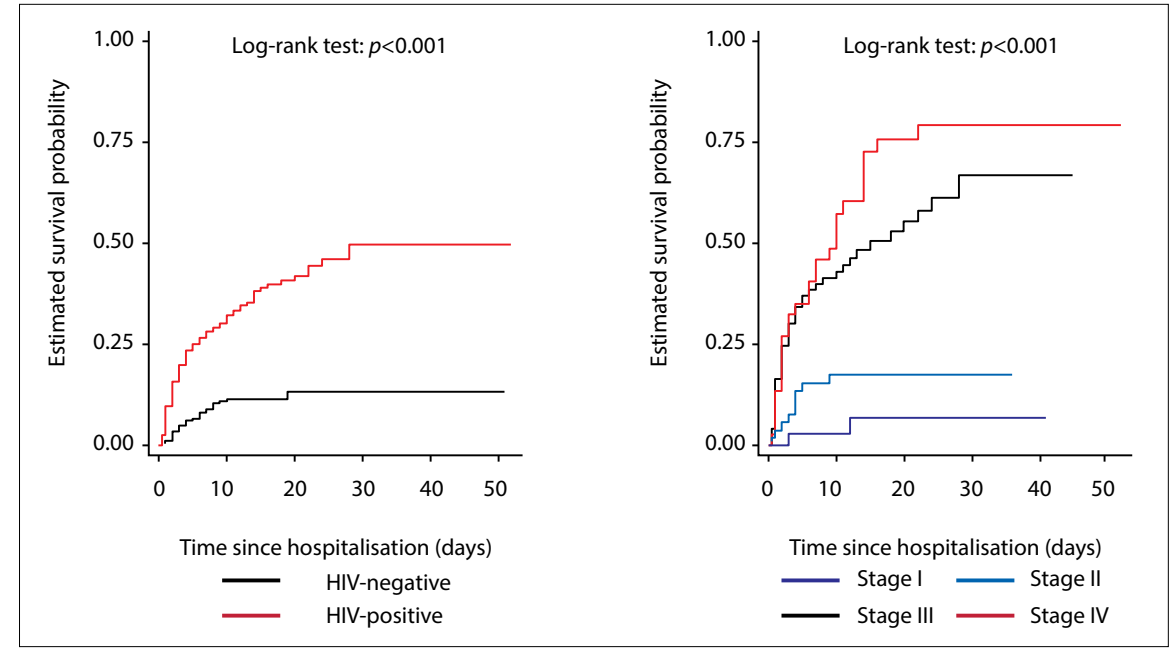

Fig. 1. Kaplan-Meier failure curves for SAM cases stratified by HIV status and HIV clinical stages.

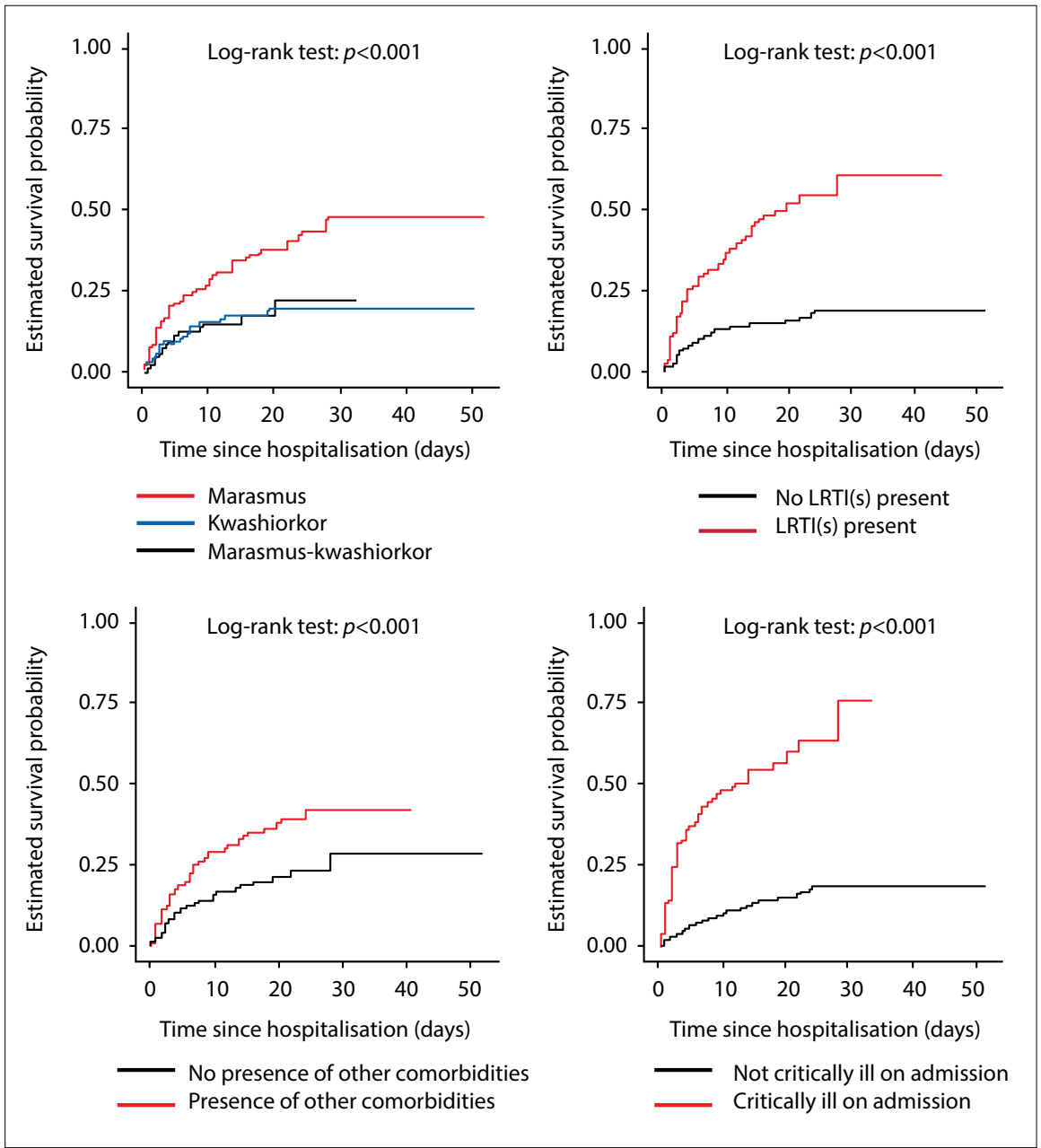

Fig. 2. Kaplan-Meier failure curves for SAM cases stratified by SAM class, presence of LRTI(s), presence of other comorbidities and critical illness.

critical illness on admission, other baseline comorbidities, LRTIs and the three SAM syndromic classifications were significantly associated with increased hazard of death. For patients with severe oedema, the hazard of death was $46 \%$ less than the hazard of dying among those who had no oedema $(p=0.001)$. Before risk factor adjustment, cases who had LRTIs had about a four times higher hazard of death compared with those who did not (HR 3.66, $p<0.001$ ). Patients admitted with other comorbidities at baseline had almost a 
two-fold higher hazard of death than those admitted without (HR 1.94, $p=0.001$ ).

HIV status and case severity were the strongest predictors of death in the unadjusted model. Children who were critically ill on admission had a five times higher hazard of death than those who were not (HR $5.70, p<0.001)$. Children who were HIV-positive and at stage IV or III of infection had, respectively, eight times higher (HR 8.12, $p<0.001$ ) and five times higher (HR 5.73, $p<0.001$ ) hazard of death than their HIV-negative counterparts. Stages II and I of HIV infection were not statistically significant predictors of mortality $(p>0.05)$.

\section{Multivariate results}

After multivariate adjustments, hospital, age, SAM syndromic classifications, dermatosis grade, other baseline comorbidities and oedema grade were not significantly associated with increased risk of death. After multivariate adjustment, the hazard rate was reduced to 1.75 for children who had LRTIs at baseline, but remained statistically significant $(p=0.001)$. HIV status and case severity were again the strongest predictors of death in adjusted models. Children who were critically ill on admission had a three-and-a-half times higher hazard of death than those who were not (HR 3.64), a result which was

Table 3. Univariate (unadjusted) and multivariate (adjusted) Cox proportional hazard models for factors associated with mortality among children with SAM during the intervention period

\begin{tabular}{|c|c|c|c|c|c|c|c|}
\hline \multirow[b]{2}{*}{ Factors } & \multirow[b]{2}{*}{$n / N$} & \multicolumn{3}{|c|}{ Unadjusted model } & \multicolumn{3}{|c|}{ Adjusted model $^{*}$} \\
\hline & & UHR & $95 \% \mathrm{CI}$ & $p$-value & AHR & 95\% CI & ${ }^{\dagger} p$-value \\
\hline \multicolumn{8}{|l|}{ Hospital } \\
\hline Hospital A & $34 / 140$ & Ref & & & Ref & & \\
\hline Hospital B & $74 / 302$ & 1.22 & $0.81-1.84$ & 0.346 & 1.55 & $0.91-1.92$ & 0.401 \\
\hline \multicolumn{8}{|l|}{ Age (months) } \\
\hline $6-12$ & $46 / 164$ & Ref & & & & & \\
\hline $13-24$ & $47 / 225$ & 0.73 & $0.49-1.11$ & 0.147 & 0.93 & $0.61-1.43$ & 0.771 \\
\hline $25-61$ & $14 / 64$ & 0.74 & $0.41-1.36$ & 0.345 & 0.61 & $0.32-1.15$ & 0.129 \\
\hline \multicolumn{8}{|l|}{ SAM classification } \\
\hline Marasmus & $63 / 173$ & Ref & & & Ref & & \\
\hline Kwashiorkor & $30 / 171$ & 0.44 & $0.29-0.69$ & $<0.001$ & 1.21 & $0.42-3.45$ & 0.806 \\
\hline M-kwashiorkor & $16 / 98$ & 0.44 & $0.25-0.77$ & 0.004 & 0.53 & $0.20-1.43$ & 0.080 \\
\hline \multicolumn{8}{|l|}{ Oedema grade } \\
\hline None & $54 / 153$ & Ref & & & Ref & & \\
\hline Mild & $6 / 29$ & 0.55 & $0.24-1.29$ & 0.171 & 0.58 & $0.22-1.53$ & 0.109 \\
\hline Moderate & $23 / 119$ & 0.52 & $0.31-0.84$ & 0.009 & 1.16 & $0.43-3.07$ & 0.675 \\
\hline Severe & $29 / 141$ & 0.46 & $0.29-0.74$ & 0.001 & 0.61 & $0.20-1.83$ & 0.682 \\
\hline \multicolumn{8}{|l|}{ Dermatosis grade } \\
\hline None & $32 / 134$ & Ref & & & Ref & & \\
\hline Mild & $23 / 102$ & 0.88 & $0.52-1.51$ & 0.652 & 0.69 & $0.37-1.26$ & 0.851 \\
\hline Moderate & $33 / 153$ & 0.90 & $0.56-1.47$ & 0.686 & 1.03 & $0.59-1.80$ & 0.439 \\
\hline Severe & $20 / 53$ & 1.73 & $0.10-1.04$ & 0.052 & 1.43 & $0.76-2.66$ & 0.088 \\
\hline \multicolumn{8}{|l|}{ LRTIs } \\
\hline No & $47 / 314$ & Ref & & & Ref & & \\
\hline Yes & $61 / 128$ & 3.66 & $2.50-5.36$ & $<0.001$ & 1.74 & $1.12-2.70$ & 0.001 \\
\hline \multicolumn{8}{|l|}{$\begin{array}{l}\text { Other baseline } \\
\text { comorbidities }\end{array}$} \\
\hline No & $56 / 294$ & Ref & & & Ref & & \\
\hline Yes & $52 / 148$ & 1.94 & $1.33-2.84$ & 0.001 & 1.14 & $0.74-1.76$ & 0.880 \\
\hline \multicolumn{8}{|c|}{ Critically ill at baseline } \\
\hline No & $44 / 328$ & Ref & & & Ref & & \\
\hline Yes & $64 / 114$ & 5.70 & $3.87-8.39$ & $<0.001$ & 3.64 & $2.35-5.64$ & $<0.001$ \\
\hline \multicolumn{8}{|l|}{ HIV status and stage } \\
\hline Negative & $29 / 250$ & Ref & & & Ref & & \\
\hline Positive/stage I & $2 / 33$ & 0.46 & $0.11-1.94$ & 0.231 & 0.20 & $0.03-1.53$ & 0.312 \\
\hline Positive/stage II & $9 / 50$ & 1.44 & $0.68-3.05$ & 0.189 & 1.12 & $0.51-2.49$ & 0.223 \\
\hline Positive/stage III & $40 / 72$ & 5.73 & $3.54-9.25$ & $<0.001$ & 3.18 & $1.85-5.47$ & $<0.001$ \\
\hline Positive/stage IV & $28 / 37$ & 8.12 & $4.82-13.66$ & $<0.001$ & 3.74 & $2.05-6.84$ & $<0.001$ \\
\hline \multicolumn{8}{|c|}{$\begin{array}{l}n / N=\text { number of failure cases over total number of cases; } \mathrm{UHR}=\text { unadjusted hazard ratio; } \mathrm{AHR}=\text { adjusted hazard ratio; Ref = reference group; } \\
\mathrm{M} \text {-kwashiorkor = marasmus-kwashiorkor. }\end{array}$} \\
\hline
\end{tabular}


statistically significant $(p<0.001)$. Severely malnourished children who were HIV-positive and at stage III or IV had, respectively, a five times higher (HR 8.12, $p<0.001$ ) and eight times higher (HR 5.73, $p<0.001)$ hazard of death than their HIV-negative counterparts. The HRs dropped by about three units for stages III and IV, but remained statistically significant $(p<0.001)$. At multivariate level, stages I and II of HIV infection remained not statistically significant predictors of mortality $(p>0.05)$.

\section{Interaction survival modelling}

Children who had LRTIs at baseline and were critically ill at the same time had a 14 times higher hazard of death compared with those who had none $(p<0.001)$ (Table 4$)$. Furthermore, children who were HIV-positive and critically ill on admission had the highest hazard of death (HR 22, $p<0.001)$ compared with those who were not exposed to any of the three risk factors. SAM cases who were HIV-positive and had LRTIs had only a nine times higher hazard of death $(p<0.001)$. A striking result was that the hazard of death was slightly lower (HR 19, $p<0.001$ ) for cases who were HIV-positive, had LRTIs and were critically ill on admission compared with those who only had HIV infection and were critically ill on admission (HR 22, $p<0.001$ ). In comparison with cases that had none of the risk factors, those who were exposed to only one of the three risk factors had lower hazards of death than those who were exposed to more than one. The predictive power of one risk factor during interaction analysis was not statistically significant $(p>0.05)$, except for critical illness $(p=0.001)$.

\section{Discussion}

This study adds an arguably new and important dimension to what already exists in the literature around the management of SAM in the context of HIV infection. In addition to HIV status itself, the study revealed independent and interactive effects of HIV disease stage, baseline disease severity and other baseline comorbidities on mortality within the context of the WHO 10-step treatment modality in two resource-limited healthcare facilities in SA.

With regard to the burden of HIV infection, the combined prevalence in both hospitals was slightly lower (43\%) than that reported in a similar and recent study conducted in SA $(51 \%),{ }^{[9]}$ but higher than that reported in a study conducted in Malawi (14\%). ${ }^{[7]}$ The current study also confirmed findings from other studies that HIV-infected SAM cases were more likely to present with marasmus than kwashiorkor on admission. ${ }^{[8,15]}$ While it is usual to present with kwashiorkor in the southern African region, with the high prevalence of HIV infection, SAM patients become wasted owing to changes in physiological and metabolic functions associated with HIV infection. ${ }^{[16]}$
This study also examined the independent effect of HIV infection on survival. The CFRs in this study were higher in the HIV-infected group (41\%) compared with their HIV-uninfected counterparts (11\%). These estimates were slightly higher than those recently reported in Burkina Faso $(39.7 \%$ v. $10.9 \%$ for HIV-infected and HIV-uninfected cases, respectively). ${ }^{[17]}$ A study in Niger also reported the same direction of relationship but with much lower CFRs for HIV-infected and HIVuninfected SAM cases (20\% and $14 \%$, respectively). ${ }^{[18]}$

In this study we have shown that disease severity at baseline was independently associated with excess mortality, a finding that is consistent with past evidence by Maitland et al.${ }^{[14]}$ Children with SAM who were admitted with one or a combination of clinical features such as coma, hypoglycaemia, hypothermia and bradycardia, had the worst survival probability compared with those who had other less SAM-related manifestations, such as herbal intoxication, Cushingoid facies, etc. These findings may have important practical implications. As Maitland et al.$^{[14]}$ have argued, the clinical features associated with higher risk of death can be used by frontline healthcare givers to target emergency treatment and allocate resources more appropriately. This is particularly important in the context of poor human and material resources in most rural hospitals in sub-Saharan Africa, such as those in which the current study was conducted. With mortality being highest in the first 5 days of admission, there is a need to institute proper, well-supported and sustained triage and emergency management mechanisms so that the 'at higher risk of death' cases are identified and treated according to the available guidelines.

Continued attention to appropriate diagnosis and treatment of common complications such as LRTIs also needs to be prioritised, as they were shown to be strongly associated with excess mortality in this study. Such complications do not always require a specialist physician and can be managed by most professional nurses in SA, with minimal supervision from a physician. It is worth noting that $\mathrm{TB}$ was found to be less common in the study sample than other LRTIs. This is probably because TB was difficult to diagnose microbiologically in the study setting, despite it being an important comorbidity in the management of SAM in the context of HIV infection. The challenge of TB diagnosis has been documented before among HIV-infected children with SAM in whom the tuberculin skin test is often falsely negative. ${ }^{[9]}$

Perhaps what the current study adds to the body of knowledge is the pattern of survival among HIV-infected SAM cases that were at different stages of HIV infection. Cox regression analyses revealed that the threshold for higher risk of death was when children with SAM were admitted at stage III. The reasons for excess mortality risk associated with advanced stages of HIV infections in SAM are

Table 4. Cox proportional hazard model for interaction of factors associated with mortality among children with SAM admitted to both hospitals during the intervention period

\begin{tabular}{|c|c|c|c|c|c|c|}
\hline \multicolumn{4}{|c|}{ Interaction terms ${ }^{*}$} & \multicolumn{3}{|c|}{ Statistics } \\
\hline HIV & $\begin{array}{l}\text { LRTI/TB at } \\
\text { baseline }\end{array}$ & $\begin{array}{l}\text { Critically ill at } \\
\text { baseline }\end{array}$ & $n / N$ & HR & $95 \%$ CI & $p$-value \\
\hline & & & $9 / 165$ & Ref & & \\
\hline 0 & 0 & 1 & $9 / 35$ & 4.83 & $1.92-12.18$ & $<0.001$ \\
\hline 0 & 1 & 0 & $3 / 35$ & 1.55 & $0.42-5.71$ & 0.532 \\
\hline 0 & 1 & 1 & $8 / 15$ & 14.64 & $5.64-38.01$ & $<0.001$ \\
\hline 1 & 0 & 0 & $12 / 89$ & 2.18 & $0.92-5.19$ & 0.411 \\
\hline 1 & 0 & 1 & $17 / 25$ & 22.00 & $9.78-49.49$ & $<0.001$ \\
\hline 1 & 1 & 0 & $20 / 39$ & 9.86 & $4.49-21.68$ & $<0.001$ \\
\hline 1 & 1 & 1 & $30 / 39$ & 19.79 & $9.39-41.73$ & $<0.001$ \\
\hline
\end{tabular}


not yet well understood, particularly in the context of antiretroviral therapy (ART) ${ }^{[19]}$ However, some authors continue to attribute excess mortality to complex pathophysiological, metabolic and pharmacological changes that occur as HIV infection progresses. ${ }^{[17]}$ HIV-infected SAM patients in the current study were initiated on ART using the standard guidelines. However, the timing of ART in preventing premature mortality among HIV-infected SAM cases at different disease stages remains a challenge. A randomised controlled trial has shown that half the children hospitalised for SAM developed oedema after starting ART. ${ }^{[20]}$ In the same study, one in 14 children who were at an advanced stage of HIV infection became oedematous 12 weeks after ART initiation.

Another contribution of this study is the demonstration of the interactive effects of multiple clinical risk factors on mortality in the study sample. A study by Koethe and Heimburger ${ }^{[21]}$ has shown that SAM, immune function and infection burden interact in children, particularly in the context of HIV infection. In this study, being critically ill and having LRTIs were, both independently and as a combination, potential effect modifiers of the higher risk of death, as they increased the hazard of death quite substantially when they were combined with HIV infection in the interaction model. The interaction model of HIV infection, LRTIs and baseline disease severity also revealed that in comparison with patients who had none of these risk factors, those who were exposed to only one of the three risk factors had lower hazards of death than those who were exposed to more than one. These findings also speak to a need to develop a triage system that helps healthcare workers to prioritise cases that are at higher risk of death.

Finally, it is also worthy to note that the combined CFR of $24.4 \%$ shown in this study is much higher than the WHO target of $<5 \%$ in the context of the WHO 10-step treatment modality, implying that achieving this target may be unrealistic, particularly among HIVinfected cases in resource-limited settings. De Maayer and Saloojee ${ }^{[9]}$ have also shown that, even in resource-privileged SA settings, the WHO target of $<5 \%$ may be impractical.

\section{Study limitations}

This study was conducted in two rural facilities to determine whether the findings would be similar. However, although we found similarities in results, these may not be generalisable beyond the study setting. There may be a layer of both internal and external facility-specific factors that determined treatment outcomes for SAM. Furthermore, the current study did not investigate how such factors may have influenced the process of care and the treatment outcomes thereof.

\section{Study recommendations}

We recommend that a carefully controlled study, such as a randomised controlled trial, be conducted to make a better judgement about the effects of the risk factors reported here on mortality in the context of the WHO treatment guidelines.

\section{Conclusion}

Within the context of increased ART and prevention of mother-tochild transmission (PMTCT) coverage in SA, there seems to be hope that the impact of HIV infection on SAM-CFRs among children may be reduced. However, given the limited evidence on the most effective ways of managing SAM patients who are HIV infected, this group of patients will continue to pose some challenges to the healthcare workforce. The high HIV prevalence rate in the study population and the resultant treatment outcomes lend support to the notion that the WHO treatment guidelines should be revised to ensure that mechanisms for effective treatment of HIV comorbidity in SAM are in place. The revisions ought to tap into the differential energy requirements by HIV status, timing of ART initiation among HIV- infected SAM cases as well as developing a broad-based triage system to more effectively identify and treat SAM cases that are at higher risk of early death. However, the target of $<5 \%$ CFR may be difficult to achieve, particularly for HIV-positive SAM cases, as has been suggested in our study findings. The role of the broader healthcare system in SA in preventing incidences of SAM at community level in the study setting is discernible. The current study has shown that the admission rates attributable to SAM at both hospitals remain high, and as such require mitigation. Community-based prevention strategies developed through multisectoral engagements have a huge role to play in achieving this goal. It goes without saying that early detection of SAM would be a crucial part of preventing disease severity and the development of multiple infections, thereby reducing the likelihood of subsequent preventable death associated with SAM in the study setting. The close linkage between SAM and HIV/AIDS begs an integrated healthcare approach to ensure that hospitals and HIV clinics work in harmony to optimise outcomes for both the child and the mother. Strengthening PMTCT services in resource-limited settings would also likely reduce the burden of HIV infection among children, and mitigate the risk of developing severe malnutrition.

Acknowledgements. The authors wish to thank the staff at the two hospitals where this study was conducted, and Prof. Ann Ashworth for her immense contribution to the conceptualisation of the research.

Funding. This study was funded by the SA National Research Foundation (NRF) and partly by the South African Centre for Epidemiological Modelling and Analysis (SACEMA).

1. Collins S, Sadler K, Dent N, et al. Key issues in the success of communitybased management of severe malnutrition. Food Nutr Bull 2006;27(3) (Suppl):S49-S70. https://doi.org/10.1177/15648265060273s304

2. World Health Organization. Management of the child with a serious infection or severe malnutrition: Guidelines for care at the first-referral level in developing countries. Geneva: WHO, 2000. http://www.who.int/child-adolescent-health/ publications/referral_care/homepage.html (accessed 14 July 2015).

3. Ashworth A, Khanum S, Jackson A, Schofield C. Guidelines for the inpatient treatment of severely malnourished children. The World Health Organization Library Cataloguing in-Publication Data 1996. Geneva: WHO, 1996.

4. Cavalcante AAM, Pinheiro LMP, Monte C, Guimaraes ARP, Ashworth A. Treatment of malnutrition in Brazil: Simple solutions to common problems. Trop Doct 1998;28(2):95-97.

5. Prudhon C, Briend A, Laurier D, Golden MH, Mary JY. Comparison of weight- and height-based indices for assessing the risk of death in severely malnourished children. Am J Epidemiol 1996;144(2):116-123. https://doi. org/10.1093/oxfordjournals.aje.a008898

6. Puoane T, Sanders D, Ashworth A, Chopra M, Strasser S, McCoy D. Improving the hospital management of malnourished children by participatory research Int J Qual Health Care 2004;16(1):31-40. https://doi.org/10.1093/intqhe/ mzh002

7. Chinkhumba J, Tomkins A, Banda T, et al. The impact of HIV on mortality during inpatient rehabilitation of severely malnourished children in Malawi. Trans R Soc Trop Med Hyg 2008;102(7):639-644. https://doi.org/10.1016/j. trstmh.2008.04.028

8. Kessler L, Daley H, Malenga G, et al. The impact of the Human immunodeficiency virus type 1 on the management of severe malnutrition in Malawi. Ann Trop Paediatr 2000;20(1):50-56. https://doi.org/10.1080/02724930092075

9. De Maayer T, Saloojee H. Clinical outcomes of severe malnutrition in a high tuberculosis and HIV setting. Arch Dis Child 2011;96(6):560-564. http:// dx.doi.org/10.1136/adc.2010.205039

10. Puoane T, Sanders D, Chopra M, et al. Evaluating clinical management of severely malnourished children: A study of two rural district hospitals. S Afr Med J 2001;91:137-141.

11. Murgod R, Ahmed M. Instant nutrition assessment in children with protein energy undernutrition. Int J Appl Bio Pharma Tech 2015;6(1)171-177.

12. World Health Organization. Improving the inpatient management of severe acute malnutrition: Toolkit to monitor current management of severe acute malnutrition. Geneva: WHO, 2010. http://www.cmamforum.org/Pool/ Resources/Toolkit-to-monitor-management-SAM-2010.pdf (accessed 12 January 2015).

13. World Health Organization. Interim WHO clinical staging of HIV/AIDS and HIV/AIDS case definitions for surveillance (African region) Geneva: WHO, 2005. http://www.who.int/hiv/pub/guidelines/casedefinitions/en/index.hml (accessed 15 January 2015). 
14. Maitland K, Berkley JA, Shebbe M, et al. Children with severe malnutrition: Can those at highest risk of death be identified with the WHO protocol? PLoS Med 2006;3(12):e500. http://dx.doi.org/10.1371/journal.pmed.0030500

15. Prazuck T, Tall F, Nacro B, et al. HIV infection and severe malnutrition: A clinical and epidemiological study in Burkina Faso. AIDS 1993;7(1)103-108. https://doi.org/10.1097/00002030-199301000-00016

16. Mehta NM, Corkins MR, Lyman B, et al. Defining paediatric malnutrition: A paradigm shift toward aetiology-related definitions. J Parenter Enteral Nutr 2013;37(4):460-481. http://dx.doi.org/10.1177/0148607113479972

17. Savadogo GL, Donnen P, Koueta F, Kafando F, Hennart P Dramaix M. Impact of HIV/AIDS on mortality and nutritional recovery among hospitalised severely malnourished children before starting antiretroviral treatment. Open J Pediatr 2013;3(4):340-345. http://dx.doi.org/10.4236/ojped.2013.34061
18. Madec Y, Germanaud D, Moya-Alvarez V, et al. HIV prevalence and impact on renutrition in children hospitalised for severe malnutrition in Niger: An argument for more systematic screening. PloS ONE 2011;6(7):e22787. http:// dx.doi.org/10.1371/journal.pone.0022787

19. Ndekha MJ, Manary MJ, Ashorn, P. Briend A. Home based therapy with ready-to-use therapeutic food is of benefit to malnourished, HIVinfected Malawian children. Acta Paediatr 2005;94(2):222-225. https://doi. org/10.1111/j.1651-2227.2005.tb01895.x

20. Prendergast A, Dangarembizi BM, Kitaka BS, et al. Hospitalisation for severe malnutrition among HIV-infected children starting antiretroviral therapy. AIDS 2011;25(7):951-956. http://dx.doi.org/10.1097/QAD.0b013e328345e56b

21. Koethe JR, Heimburger DC. Nutritional aspects of HIV-associated wasting in sub-Saharan Africa. Am J Clin Nutr 2010;91(4):1138S-1142S. http://dx.doi. org/10.3945/ajcn.2010.28608D 